\title{
Asymptotical behavior of the solution of a SDOF linear fractionally damped vibration
}

\section{system}

\author{
Z. H. Wang ${ }^{\mathrm{a}, \mathrm{b}, *}$ and M. L. Du ${ }^{\mathrm{a}}$ \\ ${ }^{\mathrm{a}}$ Institute of Science, PLA University of Science and Technology, 211101 Nanjing, China \\ ${ }^{\mathrm{b}}$ Institute of Vibration Engineering Research, Nanjing University of Aeronautics and Astronautics, 210016 \\ Nanjing, China
}

Received 3 February 2010

Accepted 8 April 2010

\begin{abstract}
Fractional-order derivative has been shown an adequate tool to the study of so-called "anomalous" social and physical behaviors, in reflecting their non-local, frequency- and history-dependent properties, and it has been used to model practical systems in engineering successfully, including the famous Bagley-Torvik equation modeling forced motion of a rigid plate immersed in Newtonian fluid. The solutions of the initial value problems of linear fractional differential equations are usually expressed in terms of Mittag-Leffler functions or some other kind of power series. Such forms of solutions are not good for engineers not only in understanding the solutions but also in investigation. This paper proves that for the linear SDOF oscillator with a damping described by fractional-order derivative whose order is between 1 and 2, the solution of its initial value problem free of external excitation consists of two parts, the first one is the 'eigenfunction expansion' that is similar to the case without fractional-order derivative, and the second one is a definite integral that is independent of the eigenvalues (or characteristic roots). The integral disappears in the classical linear oscillator and it can be neglected from the solution when stationary solution is addressed. Moreover, the response of the fractionally damped oscillator under harmonic excitation is calculated in a similar way, and it is found that the fractional damping with order between 1 and 2 can be used to produce oscillation with large amplitude as well as to suppress oscillation, depending on the ratio of the excitation frequency and the natural frequency.
\end{abstract}

Keywords: Linear oscillator, fractional-order damping, characteristic root, eigenfunction expansion

\section{Introduction}

Damping in vibrating systems occurs through the dissipation of mechanical energy, and a real system must be subject to certain level of damping. The damping in mechanical systems can be classified into three categories [1]: (i) Internal damping of material; (ii) Structural damping at joints and interfaces; (iii) Fluid damping through fluidstructure interactions. In addition, passive dampers and active dampers, two general types of external dampers, may be added to a mechanical system in order to improve its energy dissipation characteristics. Modeling damping in a dynamic system properly is very important in the utilization, analysis, and testing of the systems. In vibrating systems, the damping is usually described by a nonlinear function of the displacement and the velocity [2], and it can be simplified to a simple form under certain conditions. In the study of forced motion of a rigid plate immersed in Newtonian fluid, Bagley and Torvik derived an unusual differential equation [3].

\footnotetext{
*Corresponding author. E-mail: zhwang@ nuaa.edu.cn.
} 


$$
A \ddot{x}(t)+B_{0} D^{3 / 2} x(t)+C x(t)=f(t)
$$

The peculiarity of this equation is the fractional-order derivative $-B_{0} D^{3 / 2} x$ that is used to describe the damping force. Fractional-order derivative can be defined in different ways [4-6]. Caputo's derivative admits lots of properties that are similar to the classical derivative, thus it will be used in this paper. Let $\alpha \in \mathbb{R}, m-1<\alpha \leqslant m, m \in \mathbb{N}$, $a \in \mathbb{R}$, then Caputo's derivative is defined by

$$
{ }_{a}^{C} D_{t}^{\alpha} x(t)=\frac{1}{\Gamma(m-\alpha)} \int_{a}^{t} \frac{x^{(m)}(\tau)}{(t-\tau)^{1+\alpha-m}} d \tau .
$$

where $x^{(m)}(t)$ is the classical $m$-th derivative, and $\Gamma(z)$ is the Gamma function defined by

$$
\Gamma(z)=\int_{0}^{\infty} \mathrm{e}^{-t} t^{z-1} \mathrm{~d} t, \quad(\mathcal{R}(z)>0)
$$

satisfying $\Gamma(z+1)=z \Gamma(z)$. In the following, the notation ${ }_{a}^{C} D_{t}^{\alpha} x(t)$ will be replaced with ${ }_{a} D^{\alpha} x(t)$ for simplicity. A natural generalization of Eq. (1) is to replace ${ }_{0} D^{3 / 2} x(t)$ with ${ }_{0} D^{\alpha} x(t)$, where $0<\alpha<2$, because the term of fractional-order derivative acts also as a "damping force" so that the equilibrium is asymptotically stable if $0<\alpha<2$, as proved in [7].

In the past three decades, it has been shown that fractional-order derivative is an adequate tool to the study of so-called "anomalous" social and physical behaviors, in reflecting the non-local, frequency- and history-dependent properties of these phenomena, and dynamical systems with fractional-order derivative elements have been frequently used to model practical systems in engineering, and considerable progress has been achieved in the related topics [46,8]. For example, in [9], in the study of a passive isolation system composed of a base and two isolators, fractional-order derivatives are used to model the frequency-dependent viscoelastic isolators, and it has been found that fractional calculus model leads to better approximation of dissipated energy, and the fractional-order model requires less parameters and its fitting converges quickly. In [10], a nonlinear analysis is made for several nonlinear oscillators with elements described fractional-order derivatives, on the basis of the method of multiple scales. In general, systems involving fractional-order derivatives admit more complicated dynamics than the conventional systems. As shown in [11], for instance, when the first order derivative in the chaotic Liu system is replaced with a fractional-order derivative, chaos occurs in the fractional-order Liu system with order as low as 0.3 , and the results have been checked by circuit experiment.

A number of methods are available for solving Eq. (1) analytically or numerically. For example, the method of Laplace transform is a straightforward approach and it yields a solution in terms of the Mittag-Leffler function [4, 12]. This method, as well as the method of fractional Green's function, is the most popular method for solving linear fractional differential equations. In the various applications, however, the orders of fractional-order derivative are usually given or the solutions are expressed in general in very complicated forms. Another method is the Adomian decomposition method, a kind of method of power series [13]. This method, however, involves tedious calculation of series when a high order expansion is required. Similar to the method of eigenfunction expansion in ordinary differential equations with constant coefficients, an eigenvalue-based method assumes the solution in terms of fractional-order exponential function $[14,15]$. Such a form of solutions is very good in understanding the structure of the solutions mathematically, but it is not good in understanding their physical meanings of the solutions and the computational complexity increases sharply as the order the characteristic polynomial increases. Recently, Mark Naber proved in [16], on the basis of Laplace transform, that the solution of Eq. (1) with order $0<\alpha<1$ and $f(t)=0$ admits the following conventional form

$$
x(t)=C_{1} \mathrm{e}^{\beta t} \cos (\omega t)+C_{2} \mathrm{e}^{\beta t} \sin (\omega t)-x^{*}(t)
$$

where $\beta \pm i \omega$ are the two conjugate characteristic roots with $\beta<0, C_{1}, C_{2}$ are constants, $x^{*}(t)$ is a definite integral satisfying: $x^{*}(t)=0$ if $\alpha=1$, and $x^{*}(t) \rightarrow 0$ as $t \rightarrow+\infty$ if $0<\alpha<1$. The solution given in Eq. (3) shows clearly not only the generalization from classical vibration system to fractional vibration system, but also the difference between the vibration system with and without fractional-order derivatives. Thus, this result is helpful for engineers to understand the fractional-order dynamical systems. As mentioned in [16], the two cases $0<\alpha<1$ and $1<\alpha<2$ should be investigated separately. The main objective of this paper is to find a solution similar to Eq. (3), to show the asymptotical behavior of the free vibration, as well the steady response under harmonic excitation, for the case of $1<\alpha<2$. The three problems are discussed in Section 2, Sections 3 and Section 4 respectively, and some concluding remarks are given in Section 5. 


\section{The solution in conventional form for the fractional vibration equation}

Without loss of generality, let us consider a SDOF (single degree of freedom) fractional vibration equation in dimensionless form

$$
\ddot{x}(t)+\mu_{0} D^{\alpha} x(t)+x(t)=0, \quad(\mu>0 ; 1<\alpha<2)
$$

in the sense of Caputo's fractional-order derivative and under the following initial conditions

$$
x(0)=x_{0}, \dot{x}(0)=x_{1}
$$

Using the Laplace transform for Eq. (4) gives

$$
s^{2} X(s)-s x_{0}-x_{1}+\mu\left(s^{\alpha} X(s)-s^{\alpha-1} x_{0}-s^{\alpha-2} x_{1}\right)+X(s)=0
$$

where $X(s)=\mathcal{L}(x(t))$ is the Laplace transform of $x(t)$. Hence

$$
X(s)=\frac{s x_{0}+x_{1}+\mu\left(s^{\alpha-1} x_{0}+s^{\alpha-2} x_{1}\right)}{s^{2}+\mu s^{\alpha}+1},(1<\alpha<2)
$$

The inverse Laplace transform leads to

$$
x(t)=\frac{1}{2 \pi i} \int_{\gamma-i \infty}^{\gamma+i \infty} X(s) \mathrm{e}^{s t} \mathrm{~d} s
$$

where $\gamma>0$ is a constant.

\subsection{The characteristic roots}

In order to find the solution $x(t)$ in Eq. (6), we begin with a proof of the claim that all the roots of the following characteristic equation

$$
p(s):=s^{2}+\mu s^{\alpha}+1=0, \quad(1<\alpha<2)
$$

stay in the left-half complex plane, or equivalently, $p(s)=0$ has no roots on the imaginary axis as well as on the negative real axis (which can be easily checked) and in the right-half complex plane. In fact, let $K$ be the number of roots of $p(s)=0$ with $\mathcal{R}(s)>0$, then the Cauchy Theorem gives

$$
K=\lim _{R \rightarrow+\infty} \frac{1}{2 \pi i} \oint_{C} \frac{p^{\prime}(s)}{p(s)} \mathrm{d} s
$$

where $C=C_{1}+C_{2}$, shown in Fig. 1(a), is a closed curve (in anti-clockwise), defined by

$$
C_{1}: s=R \mathrm{e}^{\mathrm{i} \theta}, \theta \text { varies from }-\frac{\pi}{2} \text { to } \frac{\pi}{2} ; C_{2}: s=\mathrm{i} \omega, \omega \text { varies from } R \text { to }-R
$$

On $C_{1}$ with sufficient large $R$, one has

$$
\frac{p^{\prime}(s)}{p(s)}=\frac{2}{s}+O\left(\frac{1}{R^{2}}\right) \Rightarrow \frac{1}{2 \pi \mathrm{i}} \int_{C_{1}} \frac{p^{\prime}(s)}{p(s)} \mathrm{d} s \approx \frac{2}{2}=1
$$

On $C_{2}$, let $M(\omega), N(\omega), A(\omega), \phi(\omega)$ be four continuous functions satisfying

$$
\mathrm{i}^{-2} p(\mathrm{i} \omega)=M(\omega)+\mathrm{i} N(\omega)=A(\omega) e^{\mathrm{i} \phi(\omega)}
$$

where

$$
M=\omega^{2}-\mu \omega^{\alpha} \cos ((1 / 2) \alpha \pi)-1, N=-\mu \omega^{\alpha} \sin ((1 / 2) \alpha \pi)
$$

It is easy to know that $M(0)=-1<0, N(0)=0, M(+\infty)=+\infty$, and $N(\omega)<0$ as well as $M^{\prime}(\omega)>0$ for all $\omega>0$. Thus, $\phi(0)=-\pi$, and 


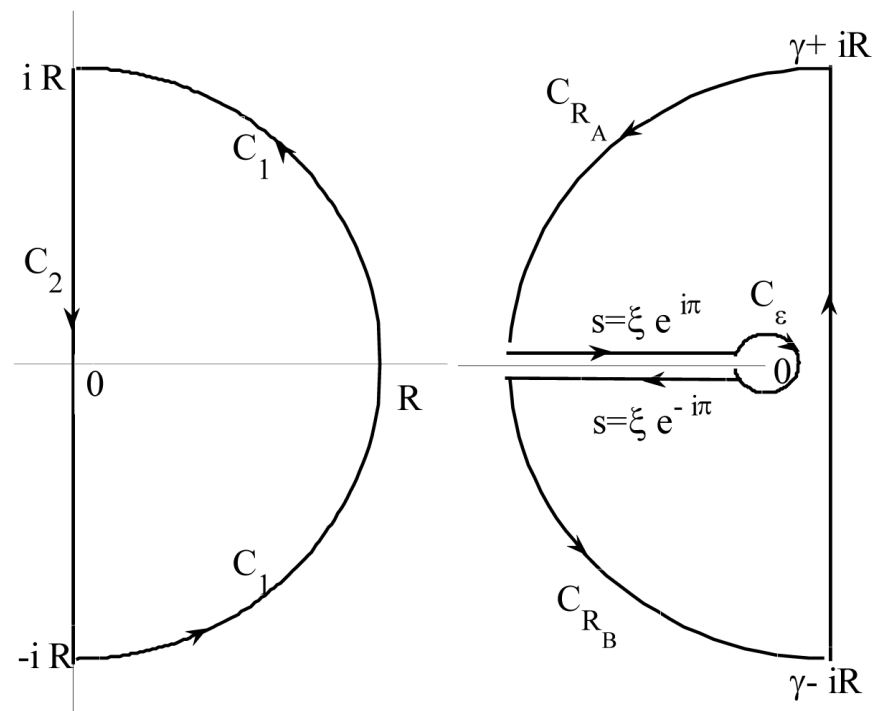

(a) The integral contour $C$

(b) The integral contour $\Gamma$

Fig. 1. The contours for integrals of complex variables.

$$
\begin{aligned}
\frac{1}{2 \pi \mathrm{i}} \int_{C_{2}} \frac{p^{\prime}(s)}{p(s)} \mathrm{d} s & =\frac{1}{2 \pi \mathrm{i}} \int_{R}^{-R}\left(\frac{A^{\prime}(\omega)}{A(\omega)}+\mathrm{i} \phi^{\prime}(\omega)\right) \mathrm{d} \omega \\
& =\frac{1}{\pi}(\phi(0)-\phi(R)) \rightarrow \frac{\phi(0)}{\pi}=-1, \quad(R \rightarrow+\infty)
\end{aligned}
$$

It follows that for all $1<\alpha<2$, one has

$$
K=1+\frac{\phi(0)}{\pi}=0
$$

That is to say, all the roots of $p(s)=0$ have negative real part.

Now, $p(s)=0$ if and only if $q(\lambda)=0$, where

$$
q(\lambda)=\lambda^{2}+\mu \lambda^{2-\alpha}+1, \lambda=\frac{1}{s}
$$

In addition,

$$
\frac{1}{a+b \mathrm{i}}=\frac{a-b \mathrm{i}}{a^{2}+b^{2}},\left(\forall a, b \in \mathbb{R}, a^{2}+b^{2} \neq 0\right)
$$

thus, $p(s)=0$ with $1<\alpha<2$ has exactly one pair of conjugate roots with negative real part if and only if $q(\lambda)$ with $0<2-\alpha<1$ has exactly one pair of conjugate roots with negative real part. For completeness, the proof of existence of the conjugated characteristic roots presented in [16] for $0<\alpha<1$ is repeated here. With $0<\alpha<1$, let $s=r \mathrm{e}^{\mathrm{i} \theta},(r>0)$, then separating the real and imaginary parts of $p(s)=0$ gives

$$
\left\{\begin{array}{l}
r^{2} \cos (2 \theta)+\mu r^{\alpha} \cos (\alpha \theta)+1=0 \\
r^{2} \sin (2 \theta)+\mu r^{\alpha} \sin (\alpha \theta)=0
\end{array}\right.
$$

When $\pi / 2<\theta<\pi$, one has $\sin (2 \theta)<0, \sin (\alpha \theta)>0$, thus, one solves uniquely from the second equation in Eq. (13) as following

$$
r=\left(\mu \frac{\sin (\alpha \theta)}{-\sin (2 \theta)}\right)^{1 /(2-\alpha)}>0
$$

Substituting it into the first equation in Eq. (13) leads to 


$$
\left(\frac{\sin ^{\alpha}(\alpha \theta)}{\sin ^{2}(2 \theta)}\right)^{1 /(2-\alpha)} \sin ((2-\alpha) \theta)=\frac{1}{\mu^{2 /(2-\alpha)}}
$$

which holds only if $\sin ((2-\alpha) \theta)>0$, namely $\frac{\pi}{2}<\theta<\frac{\pi}{2-\alpha}$. Denote the left-hand term of Eq. (14) as $\Delta(\theta)$, then

$$
\lim _{\theta \rightarrow \frac{\pi}{2}+0} \Delta(\theta)=\infty, \quad \lim _{\theta \rightarrow \frac{\pi}{2-\alpha}-0} \Delta(\theta)=0
$$

Moreover, $\Delta(\theta)$ is differentiable with respect to $\theta$ and

$$
\frac{\mathrm{d}}{\mathrm{d} \theta} \Delta(\theta)=\left(\frac{\sin ^{\alpha}(\alpha \theta)}{\sin ^{2}(2 \theta)}\right)^{1 /(2-\alpha)} \frac{\Psi}{4(2-\alpha) \sin (2 \theta) \sin (\alpha \theta)}
$$

where

$$
\begin{aligned}
\Psi= & (1-\cos (4 \theta)) \alpha^{2}+(2 \cos (4 \theta)-2 \cos (2 \alpha \theta-4 \theta) \\
& +2 \cos (2 \alpha \theta)-2) \alpha+4-4 \cos (2 \alpha \theta) \\
= & 2 \sin ^{2}(2 \theta) \alpha^{2}-8 \alpha \sin (2 \theta) \sin (\alpha \theta) \cos ((2-\alpha) \theta)+8 \sin ^{2}(\alpha \theta) \\
\geqslant & 2 \sin ^{2}(2 \theta) \alpha^{2}-8 \alpha \sin (2 \theta) \sin (\alpha \theta)+8 \sin ^{2}(\alpha \theta) \\
= & 2(\alpha \sin (2 \theta)-2 \sin (\alpha \theta))^{2}>0
\end{aligned}
$$

Because $\sin (2 \theta)<0$ and $\sin (\alpha \theta)>0$, one has

$$
\frac{\mathrm{d}}{\mathrm{d} \theta} \Delta(\theta)<0
$$

It implies that there is exactly one $\theta \in(\pi / 2, \pi /(2-\alpha))$ such that Eq. (14) holds. Similarly, Eq. (14) has exactly one root in $\theta \in(-\pi /(2-\alpha),-\pi / 2)$. Therefore, $p(s)$ has exactly one pair of conjugate complex roots only: $s_{1}=\beta+\mathrm{i} \omega-r \mathrm{e}^{i \theta}, s_{2}:=\bar{s}_{1}=\beta-\mathrm{i} \omega=r \mathrm{e}^{-i \theta}$, where $\beta<0$.

\subsection{Calculation of the contour integrals and residues}

Using the Residue Theorem for contour integral gives

$$
\begin{gathered}
x(t)+\lim _{\varepsilon \rightarrow 0, R \rightarrow+\infty}\left(\int_{C_{R_{A}}}+\int_{C_{R_{B}}}+\int_{s=\xi \mathrm{e}^{i \pi}}+\int_{s=\xi \mathrm{e}^{-i \pi}}+\int_{C_{\varepsilon}}\right) \frac{1}{2 \pi \mathrm{i}} X(s) \mathrm{e}^{s t} \mathrm{~d} s \\
=\lim _{\varepsilon \rightarrow 0, R \rightarrow+\infty} \frac{1}{2 \pi \mathrm{i}} \oint_{\Gamma} X(s) \mathrm{e}^{s t} \mathrm{~d} s=\operatorname{Res}_{s=s_{1}} X(s) \mathrm{e}^{s t}+\operatorname{Res}_{s=\bar{s}_{1}} X(s) \mathrm{e}^{s t}
\end{gathered}
$$

where $\Gamma$ is a Hankel contour encircling the two simple poles $s_{1}, s_{2}$ of $X(s)$, as shown in Fig. 1(b).

When $1<\alpha<2$, for the two simple poles $s_{1}, s_{2}=\bar{s}_{1}$ the residues are found to be

$$
\begin{aligned}
& \operatorname{Res}_{s=s_{1}} X(s) \mathrm{e}^{s t}+\operatorname{Res}_{s=\bar{s}_{1}} X(s) \mathrm{e}^{s t} \\
= & \frac{s_{1} x_{0}+x_{1}+\mu s_{1}^{\alpha-1} x_{0}+\mu s_{1}^{\alpha-2} x_{1}}{2 s_{1}+\mu \alpha s_{1}^{\alpha-1}} \mathrm{e}^{s_{1} t}+\frac{\bar{s}_{1} x_{0}+x_{1}+\mu \bar{s}_{1}^{\alpha-1} x_{0}+\mu \bar{s}_{1}^{\alpha-2} x_{1}}{2 \bar{s}_{1}+\mu \alpha \bar{s}_{1}^{\alpha-1}} \mathrm{e}^{\bar{s}_{1} t} \\
= & 2 \mathrm{e}^{\beta t} \cos (\omega t) \frac{N_{1}}{4 r^{2}+4 \alpha \mu r^{\alpha} \cos ((2-\alpha) \theta)+\alpha^{2} \mu^{2} r^{2 \alpha-2}} \\
& +2 \mathrm{e}^{\beta t} \sin (\omega t) \frac{N_{2}}{4 r^{2}+4 \alpha \mu r^{\alpha} \cos ((2-\alpha) \theta)+\alpha^{2} \mu^{2} r^{2 \alpha-2}}
\end{aligned}
$$

where $N_{1}, N_{2}$ are given as following 


$$
\begin{aligned}
N_{1}= & x_{0}\left(2 r^{2}+\alpha \mu^{2} r^{2 \alpha-2}+\mu r^{\alpha}(\alpha+2) \cos ((\alpha-2) \theta)\right) \\
& +x_{1}\left(\left(2 r+\alpha \mu^{2} r^{2 \alpha-3}\right) \cos (\theta)+\alpha \mu r^{\alpha-1} \cos ((\alpha-1) \theta)\right. \\
& \left.+2 \mu r^{\alpha-1} \cos ((\alpha-3) \theta)\right) \\
N_{2}= & x_{0} \mu r^{\alpha}(\alpha-2) \sin ((\alpha-2) \theta)+x_{1}\left(\left(2 r+\alpha \mu^{2} r^{2 \alpha-3}\right) \sin (\theta)\right. \\
& \left.+\alpha \mu r^{\alpha-1} \sin ((\alpha-1) \theta)-2 \mu r^{\alpha-1} \sin ((\alpha-3) \theta)\right)
\end{aligned}
$$

Now, we prove that the contour integrals $\int_{C_{R_{A}}} \rightarrow 0, \int_{C_{R_{B}}} \rightarrow 0$ as $R \rightarrow+\infty$, and $\int_{C_{R_{\varepsilon}}} \rightarrow 0$ if $\varepsilon \rightarrow 0$. In fact, on $C_{R_{A}}$, one has

$$
C_{R_{A}}: s=\gamma+\operatorname{Re}^{i \theta},\left(\frac{\pi}{2}<\theta<\pi\right)
$$

and $\lim _{R \rightarrow \infty} s X(s)=x_{0}$, uniformly in $\theta \in(\pi / 2, \pi)$, thus for sufficient large $R>0$, there is a $\theta$-independent-constant $Q_{1}>0$ such that

$$
|X(s)| \leqslant \frac{Q_{1}}{|s|}=\frac{Q_{1}}{\sqrt{\gamma^{2}+R^{2}+2 \gamma R \cos \theta}}
$$

Moreover, one has

$$
\lim _{R \rightarrow \infty} \frac{Q_{1}}{2 \pi} \frac{R}{\sqrt{\gamma^{2}+R^{2}+2 \gamma R \cos \theta}}=\frac{Q_{1}}{2 \pi}
$$

uniformly in $\theta \in(\pi / 2, \pi)$. Thus, there is a $\theta$-independent-constant $\hat{Q}_{1}>0$ such that for sufficient large $R>0$, one has

$$
\frac{Q_{1}}{2 \pi} \frac{R}{\sqrt{\gamma^{2}+R^{2}+2 \gamma R \cos \theta}} \leqslant \hat{Q}_{1}
$$

Note that $\gamma+R \cos (\theta)<0$ due to $\cos \theta<0$, it implies that

$$
e^{-(\gamma+R \cos (\theta)) t}>1-(\gamma+R \cos (\theta)) t
$$

Consequently one has

$$
\begin{aligned}
& \left|\int_{C_{R_{A}}} \frac{1}{2 \pi \mathrm{i}} X(s) \mathrm{e}^{s t} \mathrm{~d} s\right| \leqslant \frac{Q_{1}}{2 \pi} \int_{\pi / 2}^{\pi} \frac{\mathrm{e}^{(\gamma+R \cos (\theta)) t}}{\sqrt{\gamma^{2}+R^{2}+2 \gamma R \cos \theta}} \cdot R \mathrm{~d} \theta \\
& \leqslant \hat{Q}_{1} \int_{\pi / 2}^{\pi} \frac{1}{1-(\gamma+R \cos \theta) t} \mathrm{~d} \theta \\
& =\hat{Q}_{1} \frac{1}{a+b} \sqrt{\frac{a+b}{b-a}}\left(\ln \left|\frac{\tan \frac{\theta}{2}+\sqrt{\frac{a+b}{b-a}}}{\tan \frac{\theta}{2}-\sqrt{\frac{a+b}{b-a}}}\right|\right)_{\pi / 2}^{\pi} \rightarrow 0
\end{aligned}
$$

as $R \rightarrow+\infty$, where $a=1-\gamma t, b=-R t$. Similarly, one has $\int_{C_{R_{B}}} \rightarrow 0$ if $R \rightarrow+\infty$.

On $C_{\varepsilon}$, it holds $s=\varepsilon \mathrm{e}^{\mathrm{i} \theta},(-\pi<\theta<\pi)$, and $\lim _{\varepsilon \rightarrow 0} s^{2-\alpha} X(s)=\mu x_{1}$, uniformly in $\theta \in(\pi / 2, \pi)$. It follows that for sufficient small $\varepsilon>0$, there is a constant $Q_{2}>\stackrel{\varepsilon \rightarrow 0}{>}$ such that

$$
|X(s)| \leqslant \frac{Q_{2}}{|s|^{2-\alpha}}=Q_{2} \varepsilon^{\alpha-2}
$$

Therefore, as $\varepsilon \rightarrow 0$, one has

$$
\left|\int_{C_{\varepsilon}} \frac{1}{2 \pi \mathrm{i}} X(s) \mathrm{e}^{s t} \mathrm{~d} s\right| \leqslant \frac{Q_{2}}{2 \pi} \int_{-\pi}^{\pi} \varepsilon^{\alpha-2} e^{\varepsilon \cos (\theta) t} \cdot \varepsilon \mathrm{d} \theta \leqslant \frac{Q_{2}}{2 \pi} \int_{-\pi}^{\pi} \varepsilon^{\alpha-1} \mathrm{e}^{\varepsilon t} \mathrm{~d} \theta \rightarrow 0
$$


Special attention should be paid to the two contour integrals on the negative real axis shown in Fig. 1. The two integrals disappear for classical linear vibration equation. But for the linear fractionally damped oscillator, one has

$$
\begin{aligned}
& \left(\int_{s=\xi \mathrm{e}^{i \pi}}+\int_{s=\xi \mathrm{e}^{-i \pi}}\right) \frac{1}{2 \pi \mathrm{i}} X(s) e^{s t} \mathrm{~d} s \\
= & \frac{1}{2 \pi} \int_{\varepsilon}^{R}\left(X\left(\xi \mathrm{e}^{-\mathrm{i} \pi}\right)-X\left(\xi \mathrm{e}^{\mathrm{i} \pi}\right)\right) \mathrm{e}^{-\xi t} \mathrm{~d} \xi=\frac{1}{\pi} \int_{\varepsilon}^{R} \Im\left(X\left(\xi e^{-\mathrm{i} \pi}\right)\right) e^{-\xi t} \mathrm{~d} \xi \\
= & \frac{1}{\pi} \int_{\varepsilon}^{R} \Im\left(\frac{-\xi x_{0}+x_{1}+\mu \xi^{\alpha-1} e^{-\mathrm{i}(\alpha-1) \pi} x_{0}+\mu \xi^{\alpha-2} e^{-\mathrm{i}(\alpha-2) \pi} x_{1}}{\xi^{2}+\mu \xi^{\alpha} \mathrm{e}^{i \alpha \pi}+1}\right) \mathrm{e}^{-\xi t} \mathrm{~d} \xi \\
= & \frac{\mu}{\pi} \int_{\varepsilon}^{R} \frac{\left(\xi^{\alpha-1} x_{0}-\xi^{\alpha-2} x_{1}\right) \sin (\alpha \pi)}{\left(\xi^{2}+1\right)^{2}+2 \mu \xi^{\alpha}\left(\xi^{2}+1\right) \cos (\alpha \pi)+\mu^{2} \xi^{2 \alpha}} \mathrm{e}^{-\xi t} \mathrm{~d} \xi \\
& \rightarrow x^{*}(t):=\frac{\mu}{\pi} \int_{0}^{+\infty} \frac{\left(\xi^{\alpha-1} x_{0}-\xi^{\alpha-2} x_{1}\right) \sin (\alpha \pi)}{\left(\xi^{2}+1\right)^{2}+2 \mu \xi^{\alpha}\left(\xi^{2}+1\right) \cos (\alpha \pi)+\mu^{2} \xi^{2 \alpha}} \mathrm{e}^{-\xi t} \mathrm{~d} \xi
\end{aligned}
$$

as $\varepsilon \rightarrow 0, R \rightarrow+\infty$, where $\Im(z)$ stands for the imaginary part of $z$. As a result, the solution of the initial value problem of the fractionally damped Eq. (4) with Eq. (5) can be expressed in the form of

$$
x(t)=A \mathrm{e}^{\beta t} \cos (\omega t)+B \mathrm{e}^{\beta t} \sin (\omega t)-x^{*}(t)
$$

where the coefficients $A, B$ are given by

$$
\begin{aligned}
& A=\frac{2 N_{1}}{4 r^{2}+4 \alpha \mu r^{\alpha} \cos ((2-\alpha) \theta) \alpha^{2} \mu^{2} r^{2 \alpha-2}} \\
& B=\frac{2 N_{2}}{4 r^{2}+4 \alpha \mu r^{\alpha} \cos ((2-\alpha) \theta) \alpha^{2} \mu^{2} r^{2 \alpha-2}}
\end{aligned}
$$

It is worthy to point out that such a form of solution has been obtained early in [17] in the study of hereditary-elastic systems with weakly singular kernels, described by

$$
\ddot{x}(t)+\omega_{\infty}^{2} x(t)+\nu_{\sigma} \int_{-\infty}^{t} K\left(t-t^{\prime}\right) \ddot{x}\left(t^{\prime}\right) \mathrm{d} t^{\prime}=F\left(\delta(t)+\nu_{\sigma} K(t)\right)
$$

The integral above can be expressed in terms of fractional-order derivative if the kernel function $K(t)$ is properly chosen, as stated in [18], where a comprehensive review on the applications of fractional calculus in solid mechanics was presented.

\section{Asymptotical behavior of the solution}

\subsection{Asymptotical behavior of $x^{*}(t)$}

Denote the integrand of $x^{*}(t)$ by $h(\xi) \mathrm{e}^{-\xi t}$, then for any fixed $\eta>0$, there is a constant $Q_{3}>$ such that $|h(\xi)| \leqslant Q_{3},(\forall \xi \in[\eta, \infty))$, thus

$$
\left|\int_{\eta}^{\infty} h(\xi) \mathrm{e}^{-x i t} \mathrm{~d} \xi\right| \leqslant Q_{3} \int_{\eta}^{\infty} \mathrm{e}^{-\xi t} \mathrm{~d} \xi=\frac{Q_{3} \mathrm{e}^{-\eta t}}{t} \rightarrow 0,(t \rightarrow+\infty)
$$

In addition, $\lim _{\varepsilon \rightarrow 0} \xi^{2-\alpha} h(\xi)=\mu x_{0} \sin (\alpha \pi) / \pi$, hence there is a constant $Q_{4}>0$ such that $|h(\xi)| \leqslant Q_{4} / x i^{2-\alpha}$ for $\xi \in(0, \eta]$. Thus

$$
\left|\int_{0}^{\eta} h(\xi) \mathrm{e}^{-x i t} \mathrm{~d} \xi\right| \leqslant Q_{4} \int_{0}^{\eta} \frac{1}{\xi^{2-\alpha}}=\frac{Q_{4} \eta^{\alpha-1}}{\alpha-1} \rightarrow 0,(\eta \rightarrow 0) \rightarrow 0,(t \rightarrow+\infty)
$$

Consequently, for any given $\varepsilon>$, there is a $\eta>0$ such that 


$$
\left|\int_{0}^{\eta} h(\xi) \mathrm{e}^{-\xi t} \mathrm{~d} \xi\right|<\frac{\varepsilon}{2}
$$

and with this $\eta>0$, there is a constant $T>0$ such that

$$
\left|\int_{\eta}^{\infty} h(\xi) \mathrm{e}^{-\xi t} \mathrm{~d} \xi\right|<\frac{\varepsilon}{2},(t>T)
$$

Thus for $t>T$, it holds

$$
\left|x^{*}(t)\right| \leqslant\left|\int_{0}^{\eta} h(\xi) \mathrm{e}^{-\xi t} \mathrm{~d} \xi\right|+\left|\int_{\eta}^{\infty} h(\xi) \mathrm{e}^{-\xi t} \mathrm{~d} \xi\right|<\varepsilon
$$

This means that for all $1<\alpha<2$ and $\mu>0$, one has

$$
\lim _{t \rightarrow+\infty} x^{*}(t)=0
$$

Together with the result proved in [16], one concludes that for the linear oscillator defined in Eq. (4) with $0<\alpha<2$, the solution of its initial value problem consists of two parts, the first one is $A \mathrm{e}^{\beta t} \cos (\omega t)+B \mathrm{e}^{\beta t} \sin (\omega t)$ that is similar to the case without fractional-order derivative, and the second one is a definite integral $x^{*}(t)$ that is independent of the two characteristic roots, which decays to 0 as $t \rightarrow+\infty$ and disappears for the classical linear oscillator.

As seen above, for $\mu>0$ and $0<\alpha<2$, one has $x(t) \rightarrow 0$ if $t \rightarrow+\infty$. Thus, the trivial solution $x=0$ of Eq. (4) is asymptotically stable for $\mu>0$ and $0<\alpha<2$. That is to say, the asymptotical stability of $x=0$ of Eq. (4) can be justified if all the characteristic roots have negative real parts, just as in the stability theory of ordinary differential equations. For Eq. (4), the second equation in Eq. (13) does not hold in the cases of $\theta=\pi, \theta= \pm \pi / 2$, $0<\theta<\pi / 2$ and $-\pi / 2<\theta<0$, it implies that all the roots of $p(s)=s^{2}+\mu s^{\alpha}+1$ have negative real parts. In Section 2.1 of this paper, this fact is confirmed intentionally in an indirect way by using the Cauchy Theorem, because this idea, reported in [19] for delay differential equations, can be used to check the asymptotical stability of some more general differential equations with fractional-order derivatives.

\subsection{Asymptotical behavior of the solution $x(t)$}

The two parts $\hat{x}(t)=A \mathrm{e}^{\beta t} \cos (\omega t)+B \mathrm{e}^{\beta t} \sin (\omega t)$ and $x^{*}(t)$ consist of the solution of the fractional vibration equation. Though they decay to zero respectively as $t \rightarrow+\infty$, the decaying ratios of $x^{*}(t)$ and $\hat{x}(t)$ are of not the same order, $x^{*}(t)$ approaches to zero much faster than $\hat{x}(t)$. In fact, the first part $\hat{x}(t)$ oscillates with the amplitude $\sqrt{A^{2}+B^{2}} \mathrm{e}^{\beta t}$, which admits the maximum $\sqrt{A^{2}+B^{2}}$ at $t=0$. As for the integral $x^{*}(t)$, it is bounded by $\tilde{x}(t)$ :

$$
\left|x^{*}(t)\right| \leqslant \tilde{x}(t):=\frac{\mu}{\pi} \int_{0}^{+\infty} \frac{\left(\xi^{\alpha-1}\left|x_{0}\right|+\xi^{\alpha-2}\left|x_{1}\right|\right)|\sin (\alpha \pi)|}{\left(\xi^{2}+1\right)^{2}+2 \mu \xi^{\alpha}\left(\xi^{2}+1\right) \cos (\alpha \pi)+\mu^{2} \xi^{2 \alpha}} \mathrm{e}^{-\xi t} \mathrm{~d} \xi
$$

It is easy to see that $\sqrt{A^{2}+B^{2}} \mathrm{e}^{\beta t}$ and $\tilde{x}(t)$ decay monotonically to zero. Moreover, for $t>>1$, one has

$$
\begin{aligned}
& \frac{\left|\tilde{x}^{\prime}(t)\right|}{\mathrm{e}^{\beta t}}=\frac{\mu}{\pi} \int_{0}^{+\infty} \frac{\xi\left(\xi^{\alpha-1}\left|x_{0}\right|+\xi^{\alpha-2}\left|x_{1}\right|\right)|\sin (\alpha \pi)|}{\left(\xi^{2}+1\right)^{2}+2 \mu \xi^{\alpha}\left(\xi^{2}+1\right) \cos (\alpha \pi)+\mu^{2} \xi^{2 \alpha}} \mathrm{e}^{-(\xi+\beta) t} \mathrm{~d} \xi \\
&=\frac{\mu}{\pi}\left(\int_{0}^{-\beta}+\int_{-\beta}^{+\infty}\right) \frac{\xi\left(\xi^{\alpha-1}\left|x_{0}\right|+\xi^{\alpha-2}\left|x_{1}\right|\right)|\sin (\alpha \pi)|}{\left(\xi^{2}+1\right)^{2}+2 \mu \xi^{\alpha}\left(\xi^{2}+1\right) \cos (\alpha \pi)+\mu^{2} \xi^{2 \alpha}} \mathrm{e}^{-(\xi+\beta) t} \mathrm{~d} \xi \\
&>\frac{\mu}{\pi} \int_{0}^{-\beta} \frac{\xi\left(\xi^{\alpha-1}\left|x_{0}\right|+\xi^{\alpha-2}\left|x_{1}\right|\right)|\sin (\alpha \pi)|}{\left(\xi^{2}+1\right)^{2}+2 \mu \xi^{\alpha}\left(\xi^{2}+1\right) \cos (\alpha \pi)+\mu^{2} \xi^{2 \alpha}} \mathrm{e}^{-(\xi+\beta) t} \mathrm{~d} \xi \\
&=\mathrm{e}^{-(k+\beta) t} \cdot \frac{\mu}{\pi} \int_{0}^{-\beta} \frac{\xi\left(\xi^{\alpha-1}\left|x_{0}\right|+\xi^{\alpha-2}\left|x_{1}\right|\right)|\sin (\alpha \pi)|}{\left(\xi^{2}+1\right)^{2}+2 \mu \xi^{\alpha}\left(\xi^{2}+1\right) \cos (\alpha \pi)+\mu^{2} \xi^{2 \alpha}} \mathrm{e}^{-(\xi+\beta) t} \mathrm{~d} \xi \\
&>>|\beta| \sqrt{A^{2}+B^{2}},(1<\alpha<2)
\end{aligned}
$$


where $-(\kappa+\beta)>0$ and $\kappa \in(0,-\beta)$ is a fixed number due to the mean-value theorem for integral. It means that $\tilde{x}(t)$ decay to zero much faster than $\sqrt{A^{2}+B^{2}} \mathrm{e}^{\beta t}$. A similar inequality can be obtained also for $0<\alpha<1$. As a result, the integral $x^{*}(t)$ in the solution of the fractionally damped oscillator can be neglected when one focuses on the stationary solution of the fractional vibration equation, namely for $0<\alpha<2$ with $\alpha \neq 1$, one has

$$
x(t) \approx A \mathrm{e}^{\beta t} \cos (\omega t)+B \mathrm{e}^{\beta t} \sin (\omega t),(t>>1)
$$

a form that we get used to in the theory of ordinary differential equations. It should be noted, however, that $e^{(\beta+i \omega) t} c$ is not a solution of Eq. (4), due to

$$
{ }_{0} D^{\alpha} \mathrm{e}^{\lambda t} \neq \lambda^{\alpha} \mathrm{e}^{\lambda t}
$$

\section{Steady state of the harmonic response}

The response can be calculated in a similar way given in Section 2, if the fractionally damped oscillator is subjected to external excitation. For example, if the excitation is harmonic, say, $\sin (\Omega t)$,

$$
\ddot{x}(t)+\mu_{0} D^{\alpha} x(t)+x(t)=\sin (\Omega t),(\mu>0 ; 1<\alpha<2)
$$

then the response under the zero initial condition $x(0)=0, \dot{x}(0)=0$ has the following form

$$
x(t)=x_{f}(t):=\frac{1}{2 \pi \mathrm{i}} \int_{\gamma-\mathrm{i} \infty}^{\gamma+i \infty} \frac{\Omega \mathrm{e}^{s t}}{\left(s^{2}+\Omega^{2}\right)\left(s^{2}+\mu s^{\alpha}+1\right)} \mathrm{d} s
$$

As done in Section 2, one can find that $x_{f}(t)$ has a similar form as Eq. (18), namely

$$
x_{f}(t)=\hat{A} \mathrm{e}^{\beta t} \cos (\omega t)+\hat{\beta} \mathrm{e}^{\beta t} \sin (\omega t)-x_{f}^{*}+a \cos (\Omega t)+b \sin (\Omega t)
$$

where $\hat{A} \mathrm{e}^{\beta t} \cos (\omega t)+\hat{B} \mathrm{e}^{\beta t} \sin (\omega t), x_{f}^{*}(t)$ decay to zero as $t \rightarrow+\infty$, and $a, b$ are two constants. Therefore, for $t>>1$, one has

$$
x_{f}(t) \approx x_{s}(t):=a \cos (\Omega t)+b \sin (\Omega t)=F \sin (\Omega t-\phi)
$$

where the amplitude $F$ and the phase $\phi$ are given by

$$
\begin{aligned}
& F=\frac{1}{\sqrt{\Omega^{4}+\mu^{2} \Omega^{2 \alpha}-2 \Omega^{2}+2 \mu \Omega^{\alpha}\left(-\Omega^{2}+1\right) \cos \left(\frac{\alpha \pi}{2}\right)+1}} \\
& \phi=\arctan \frac{\mu \Omega^{\alpha} \sin \left(\frac{\alpha \pi}{2}\right)}{-\Omega^{2}+\mu \Omega^{\alpha} \cos \left(\frac{\alpha \pi}{2}\right)+1}
\end{aligned}
$$

Figure 2 presents a number of case studies, showing that the steady solution $x_{s}(t)$ given in Eq. (26) matches the solution $x_{f}(t)$ given in Eq. (25) of the fractionally damped oscillator perfectly.

The order of the fractional-order derivative has important effect on the steady periodic solution. As seen in Fig. 3, for fixed $\alpha$ in the nearby of 0 or 2, the amplitude has a peak when the ratio of the excitation frequency and the natural frequency is close to 1 if $\mu \in(0,1)$ is small. These cases are similar to the undamped oscillator under harmonic excitation, thus resonance occurs as is expected. And for fixed $\mu>0$, the damping of fractional-order between 1 and 2 produces oscillation with larger amplitude than that of fractional-order between 0 and 1 . When the ratio of the excitation frequency and the natural frequency is less than 1 , the fractional damping with order $1<\alpha<2$ can be used to produce oscillation with larger amplitude, and when the ratio of the excitation frequency and the natural frequency is greater than 1 , the fractional damping with order $1<\alpha<2$ can be used to suppress oscillation. 

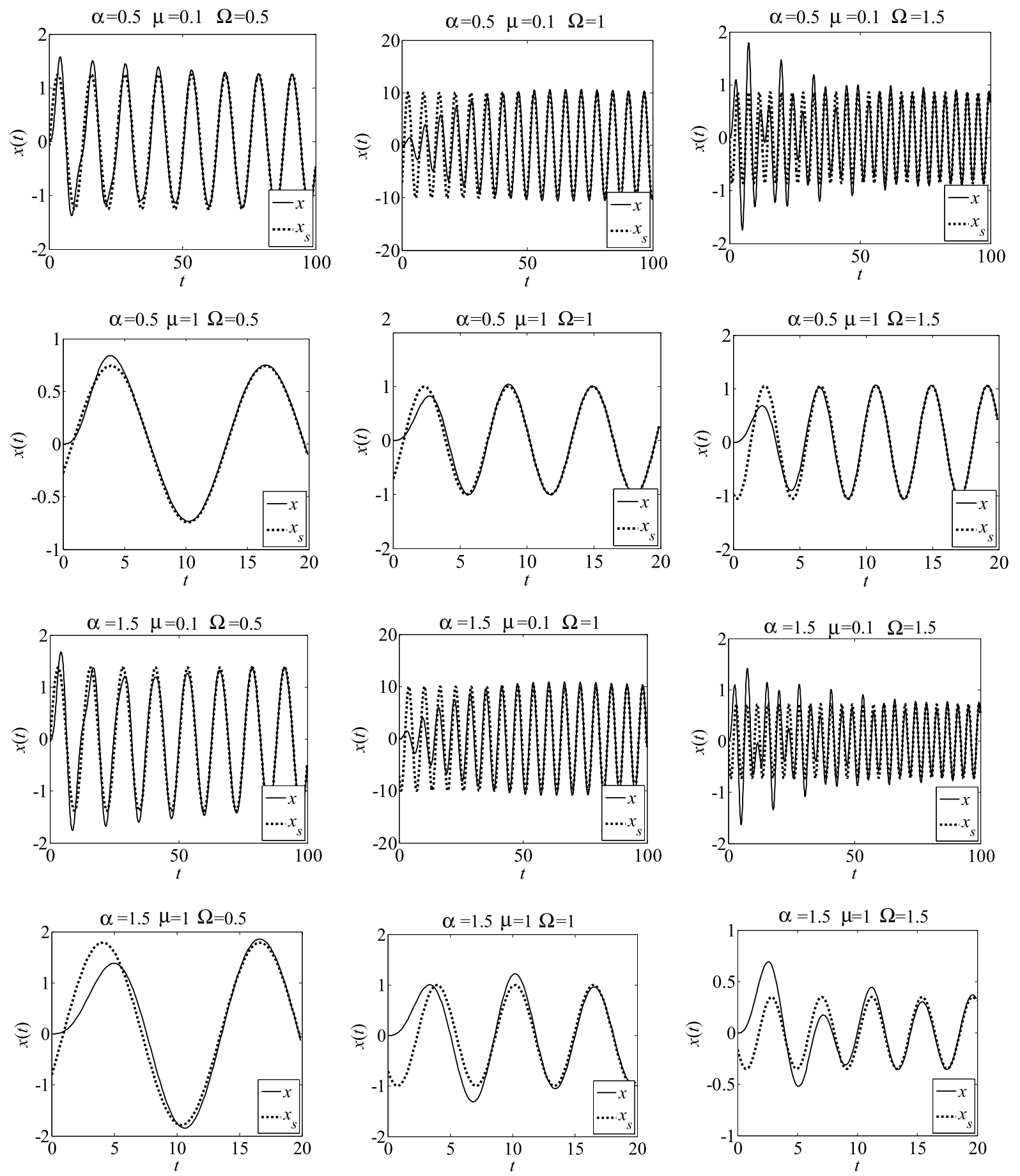

Fig. 2. Comparison of the harmonic response: -numerical solution; -steady solution. 

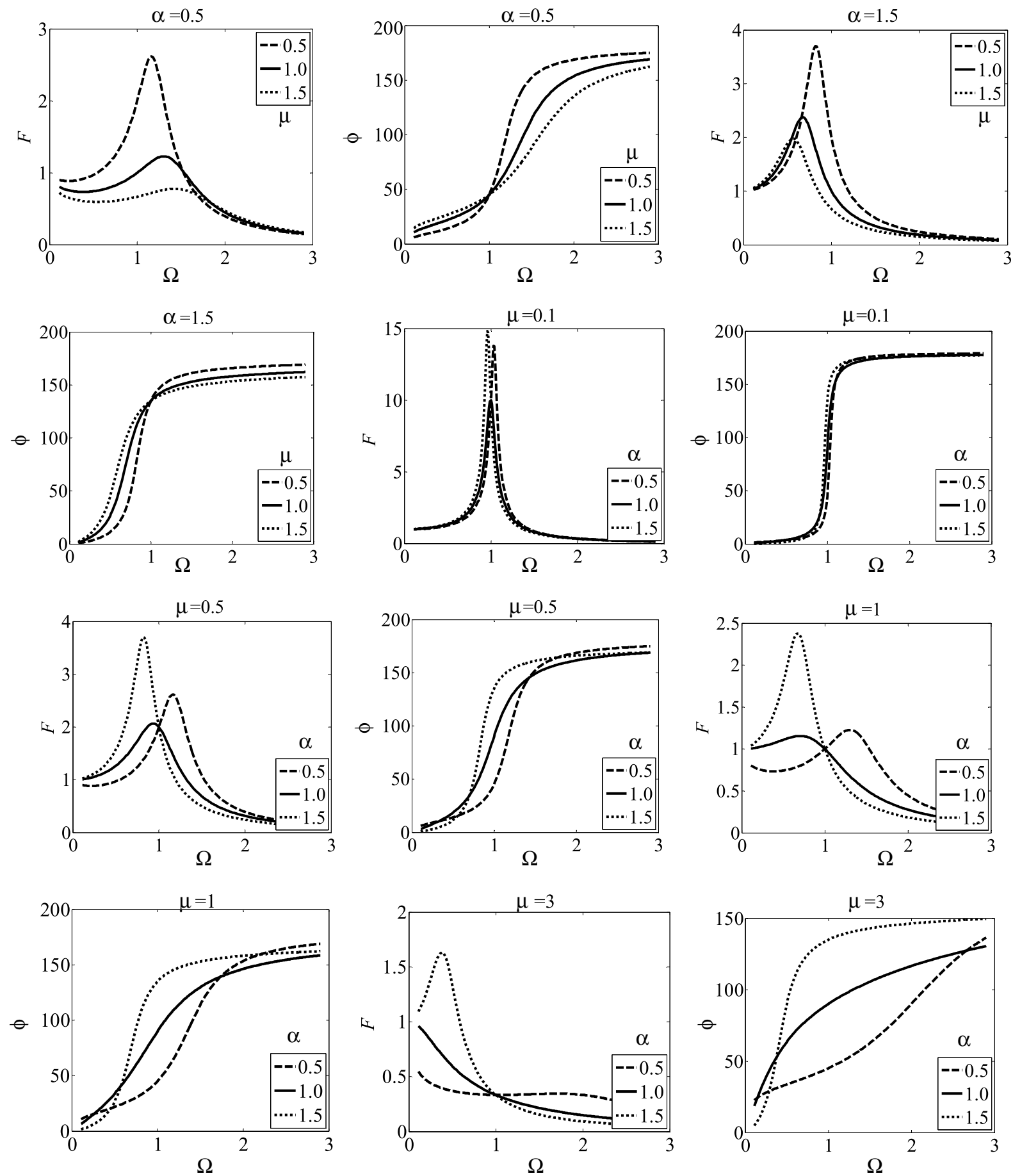

Fig. 3. The steady response for different and different. 


\section{Conclusions}

The fractional calculus has a history nearly as long as the classical calculus, but it is still not popular in engineering applications. One reason is probably that the results are not expressed in forms that are easy to understand for engineers. This paper focuses on the asymptotical properties of the solution of a SDOF linear oscillator with damping described by fractional-order derivative of order between 1 and 2. For the free vibration, it is shown that the solution of its initial value problem consists of two parts in conventional forms that are easy to understand for engineers, the first one is the 'eigenfunction expansion' associated with the characteristic roots, a case similar to the one for systems without fractional-order derivative, and the second one is a definite integral that is independent of the characteristic roots, and the stationary solution can be dominated by the "eigenfunction expansion" only. For the forced vibration under harmonic excitation, the response decays to the steady periodic motion rapidly. The order of the fractional-order derivative has important effect on the steady periodic solution, the damping of fractional-order between 1 and 2 produces oscillation with larger amplitude than that of fractional-order between 0 and 1 . The fractional damping with order between 1 and 2 can be used to produce oscillation with large amplitude as well as to suppress oscillation, depending on the ratio of the excitation frequency and the natural frequency.

\section{Acknowledgements}

The first author would like to thank the financial support of the National Natural Science Foundation of China under Grant 10825207, and to thank the organizers of the International Conference on Vibro-Impact Systems 2010 (ICoVIS2010) for giving him an opportunity to preset these results.

\section{References}

[1] C. W. de Silva, Vibration Damping, In: de Silva C. W. (Ed),Vibration Damping, Control, and Design, Boca Raton: CRC Press, 2007.

[2] M. Borowiec, G. Litak and A. Syta, Vibration of the Duffing oscillator: Effect of fractional damping, Shock and Vibration 14 (2007), 29-36.

[3] R.L. Bagley and P.J. Torvik, On the appearance of the fractional derivative in the behavior of real materials, ASME Journal of Applied Mechanics 51 (1984), 294-298.

[4] I. Podlubny, Fractional Differential Equations, San Diego: Academic Press, 1999.

[5] A.A. Kilbas, H.M. Srivastava and J.J. Trujillo, Theory and Applications of Fractional Differential Equations, Amsterdam: Elsevier, 2006.

[6] S. Das, Functional Fractional Calculus for System Identification and Controls, Berlin: Springer-Verlag, 2008.

[7] Z.H. Wang and H.Y. Hu, Stability of a linear oscillator with damping force of fractional-order derivative, Science China: Physics, Mechanics \& Astronomy 53 (2010), 345-352.

[8] J. Sabatier, O.P. Agrawal abd J.A. Tenreiro Machado, eds, Advances in Fractional Calculus, Dordrecht: Springer, 2007.

[9] A. Coronado, M.A. Trindade and R. Sampaio, Frequency-dependent viscoelastic models for passive vibration isolation systems, Shock and Vibration 9 (2002), 253-264.

[10] Y.A. Rossikhin and M.V. Shitikova, New approach for the analysis of damped vibrations of fractional oscillators, Shock and Vibration 16 (2009), 365-387.

[11] X.R. Chen, C.X. Liu, F.Q. Wang and Y.X. Li, Study on the fractional-order Liu chaotic system with circuit experiment and its control, Acta Physica Sinica 57 (2007), 1416-1422. (In Chinese).

[12] L.E. Suarez and A. Shokooh, An eigenvector expansion method for the solution of motion containing fractional derivatives, ASME Journal of Applied Mechanics 64 (1997), 629-635.

[13] Ray S. Saha, B.P. Poddar and R.K. Bera, Analytical solution of a dynamic system containing fractional derivative of order one-half by Adomian decomposition method, ASME Journal of Applied Mechanics 72 (2005), 290-295.

[14] B. Bonilla, M. Rivero and J.J. Trujillo, Linear differential equations of fractional orders, in: Advances in Fractional Calculus, J. Sabatier, O.P. Agrawal and J.A. Tenreiro, Machado, eds, Dordrecht: Springer, 2007, pp. 77-91.

[15] Z.H. Wang and X. Wang, General solution of the Bagley-Torvik equation with fractional-order derivative, Communications in Nonlinear Science and Numerical Simulation 15 (2010), 1279-1285.

[16] M. Naber, Linear fractionally damped oscillator, International Journal of Differential Equations, Volume 2010, Article ID 197020, 12 pages, doi:10.1155/2010/197020.

[17] V.M. Zelenev, S.I. Meshkov and Yu. A. Rossikhin, Damped vibrations of hereditary-elastic systems with weakly singular kernels, Journal of Applied Mechanics and Technical Physics 11 (1970), 290-293.

[18] Yu. A. Rossikhin and M.V. Shitikova, Application of fractional calculus for dynamic problems of solid mechanics: Novel trends and recent results, Applied Mechanics Reviews 63 (2010), 010801-1 52.

[19] B.D. Hassard, Counting roots of the characteristic equation for linear delay-differential systems, Journal of Differential Equations 136 (1997), 222-235. 

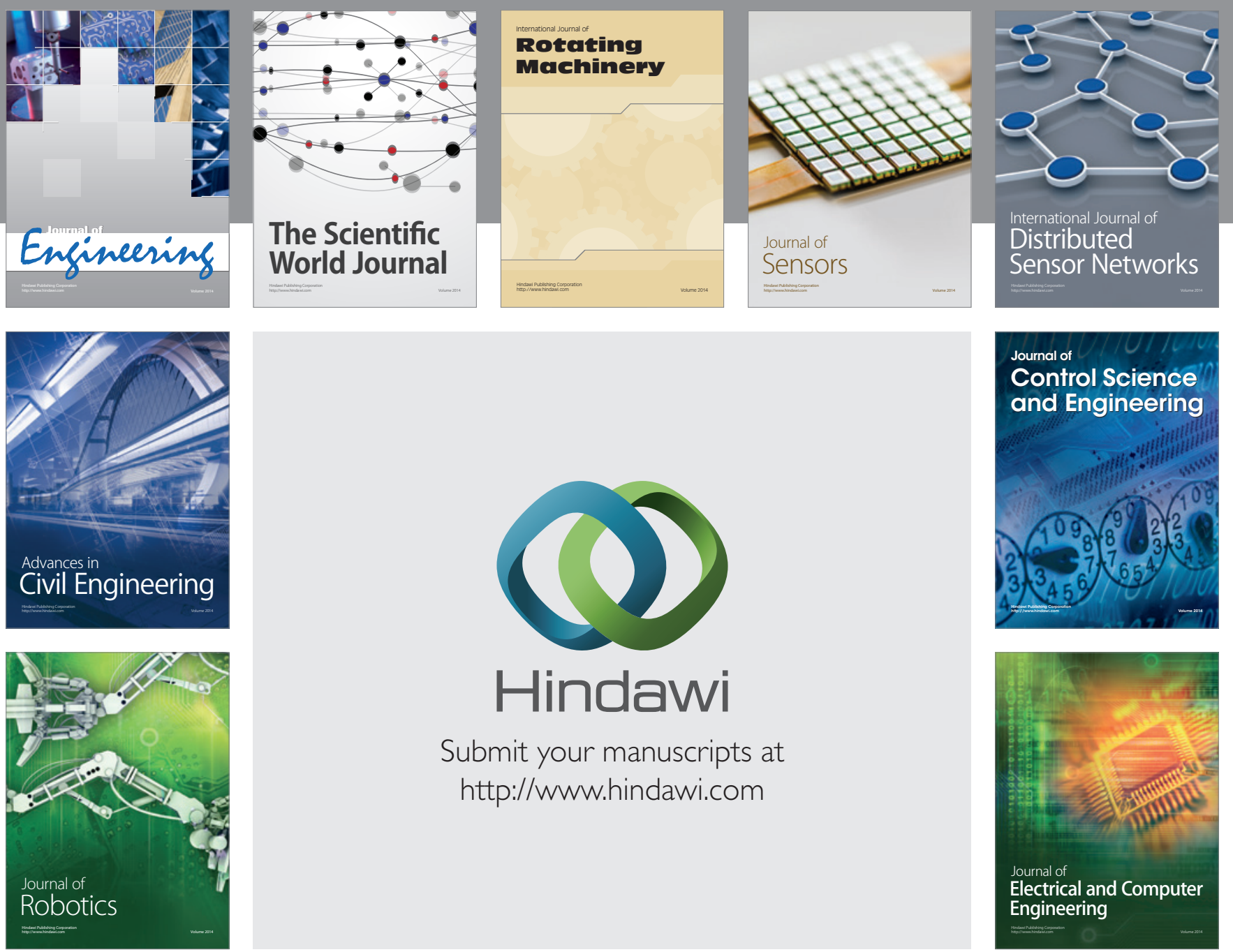

Submit your manuscripts at

http://www.hindawi.com
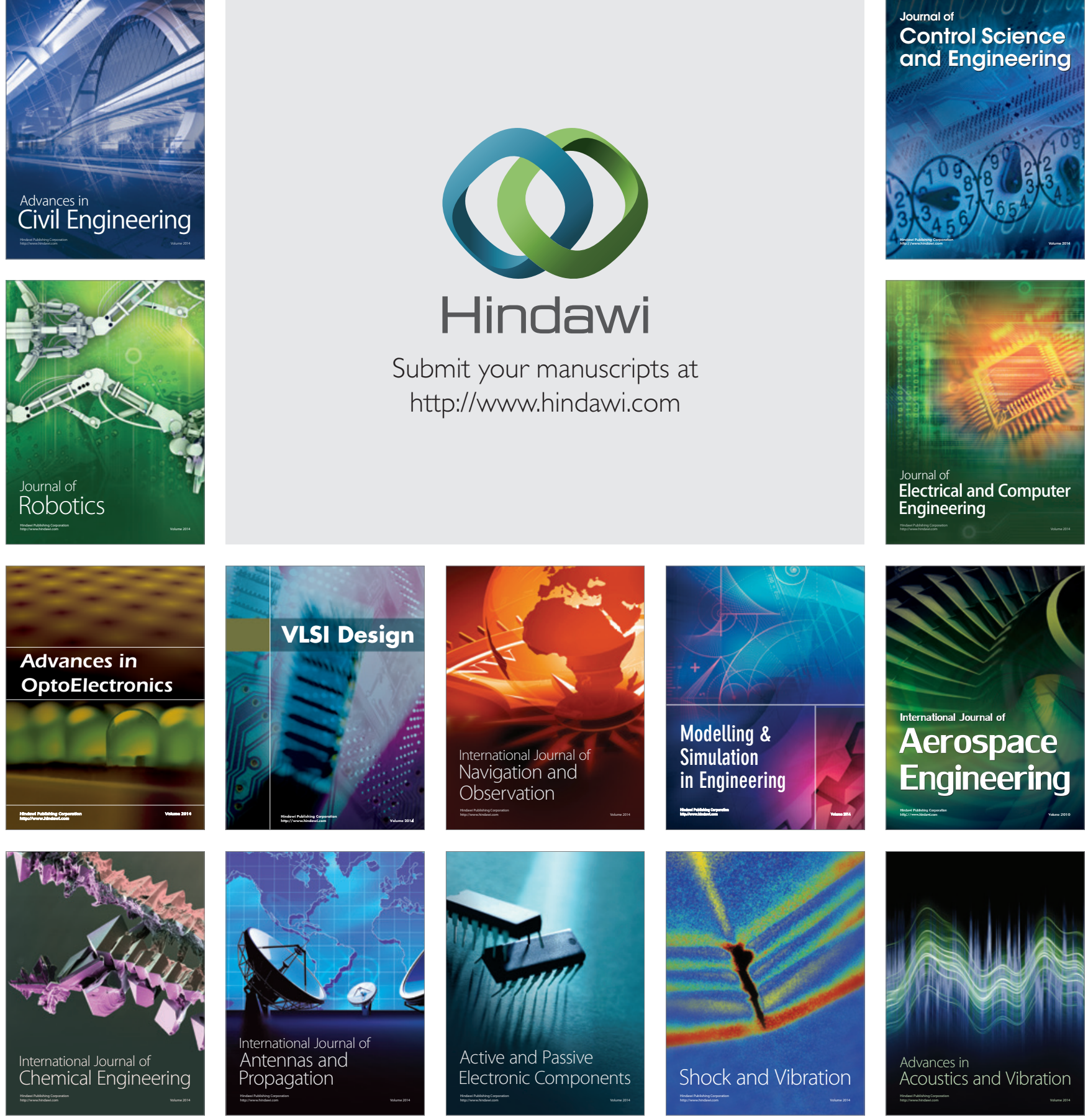